\title{
Комбинационное рассеяние света квазиоднофотонных импульсов в оптоволокне с накачкой
}

\author{
(C) В.Г. Попов ${ }^{1,2}$, В.Г. Криштоп ${ }^{1,2}$, С.А. Тарелкин ${ }^{3}$, И.И. Корель ${ }^{4}$ \\ ${ }^{1}$ Центр научных исследований и перспективных разработок, ОАО „ИнфоТеКС“, \\ 127287 Москва, Россия \\ ${ }^{2}$ Институт проблем технологии микроэлектроники Российской академии наук, \\ 142432 Черноголовка, Московская обл., Россия \\ ${ }^{3}$ Всероссийский научно-исследовательский институт оптико-фризических измерений, \\ 119361 Москва, Россия \\ ${ }^{4}$ Новосибирский государственный технический университет (физико-технический фракультет), \\ 630073 Новосибирск, Россия \\ E-mail: Vladimir.Popov@infotecs.ru
}

Поступила в Редакцию 15 апреля 2020 г.

В окончательной редакции 21 апреля 2020 г.

Принята к публикации 21 апреля 2020 г.

Теоретически рассмотрены процессы комбинационного рассеяния света квазиоднофотонных импульсов в одномодовом оптическом волокне с накачкой. Особенность рассеяния в том, что накачка создает неравновесные молекулярные колебания в оптоволокне, что существенно увеличивает вероятность комбинационного рассеяния света. Неравновесные колебания ожидаются в ситуации, когда для импульса накачки реализуется режим стимулированного комбинационного рассеяния света. В результате получены оценки длины оптоволоконной линии с повышенной вероятностью комбинационного рассеяния света.

Ключевые слова: комбинационное рассеяние, оптоволокно, фононы.

DOI: 10.21883/FTP.2020.08.49631.07

\section{1. Введение}

Комбинационное рассеяние света (КРС) в веществе было открыто в 30-х годах XX века независимо в России Мандельштамом (МГУ) [1] и в Индии Раманом (Университет Калькутты) [2]. С тех пор комбинационное рассеяние света активно используется в спектроскопии различных веществ [3]. В оптоволоконных системах активно используется индуцированное или комбинационное (рамановское) рассеяние света [4], на основе которого создаются рамановские лазеры и усилители [5]. Стимулированное комбинационное рассеяние света это сугубо нелинейный эффект, обусловленный индуцированием стоксового перехода фотонами в оптоволоконной линии. Примечательно, что такое индуцированное излучение повышает вероятность комбинационного рассеяния света от $10^{-6}$ до $10^{-1}$, т.е. на 5 порядков от величины спонтанного рассеяния [6]. Такое резкое увеличение вероятности, с одной стороны, обусловлено большим числом фотонов в волокне, а с другой следует ожидать когерентное индуцированное излучение и в фононной подсистеме. Оптические фононы (более точно - поляритоны) в отличие от фотонов практически неподвижны и сохраняют свои состояния продолжительное время после прохождения импульса накачки $(\tau \sim 2.1$ пс, как можно определить по полуширине стоксовых пиков в спектрах КРС [7]). Такие фононные колебания можно использовать для усиления КРС слабых фотонных импульсов, актуальность которых резко возросла в связи с их использованием в устрой- ствах квантового распределения ключей в квантовой криптографии [8].

На сегодняшний день КРС в оптическом волокне подробно исследуется не только с целью повышения качества оптоволоконных усилителей, но и для формирования излучения со сложной спектральной структурой типа суперконтинуума [9]. При этом используется накачка мощными ультракороткими пикосекундными импульсами (70 пс), что приводит не только к излучению суперконтинуума, но и каскадному КРС [10]. Наблюдение такого каскада свидетельствует о значительно большем времени релаксации оптических фононов или молекулярных колебаний в волокне $(\tau>70$ пс) и дает возможность их практического использования.

В данной работе предложена теоретическая модель, рассматривающая КРС в существенно неравновесной ситуации, когда фононные колебания в волокне значительно перегреты комбинационным рассеянием импульса накачки. В следующем разделе мы опишем модель и ограничения, в рамках которых рассматривается неравновесное КРС. В 3 разделе мы обсудим упрощение и получение количественных оценок для длины оптического волокна. В заключение будут сформулированы выводы и обсуждено дальнейшее развитие данного подхода для устройств преобразования энергии излучения в оптоэлектронных устройствах.

\section{2. Описание модели}

В данной работе используется дипольное приближение для описания интенсивности $W$ КРС фотонов на фо- 
нонах. В этом случае имеем следующее выражение [11]:

$$
W \sim \omega^{4}(d \varepsilon / d u)^{2}\left\langle u_{02}\right\rangle,
$$

где $\omega-$ частота фотона, $\varepsilon-$ поляризуемость, $u-$ смещение атомов, $\left\langle u_{02}\right\rangle-$ средний квадрат амплитуды смещения атомов. В ситуации, когда частота фононов $\omega_{0}$ много меньше частоты фотона, т. е. $\omega \gg \omega_{0}$, средняя амплитуда может быть представлена как сумма стоксового и антистоксового членов:

$$
\begin{aligned}
\left\langle u_{02}\right\rangle & =\left|\left\langle n+1\left|u_{0}\right| n\right\rangle\right|^{2}+\left|\left\langle n-1\left|u_{0}\right| n\right\rangle\right|^{2} \\
& =\hbar(2 n+1) /\left(4 M N \omega_{0}\right),
\end{aligned}
$$

где $M$ - приведенная масса атомов, $N$ - число элементарных ячеек, $n-$ число фононов, $\hbar-$ постоянная Планка

$$
W \sim \frac{\omega^{4}(d \varepsilon / d u)^{2}}{4 M N \omega_{0}} \hbar(2 n+1) .
$$

В равновесной ситуации $n=n_{0}=1 /\left(\exp \left(\hbar \omega_{0} / k T\right)-1\right)$, где $k-$ постоянная Больцмана, $T$ - температура. В случае же накачки $n_{r} \sim I_{r}(z)$, где $I_{r}(z)-$ интенсивность стоксового излучения, возникшего при накачке оптоволоконной линии. Зависимость интенсивности от координаты вдоль оптоволоконной линии возникает вследствие затухания импульса накачки из-за рассеяния, в том числе из-за стимулированного КРС. Так как индуцированное КРС имеет пороговый характер, существует и порог для интенсивности стоксового излучения $I_{\text {rpt}}$, ниже которого ожидать когерентности фононных колебаний не приходится. Расстояние $L$, на котором $I_{\mathrm{rp}}(L)=I_{\mathrm{rpt}}$, и определяет оптимальную длину оптоволоконной линии, которую будем называть длиной стимулированного комбинационного рассеяния света (СКРС).

Таким образом, решающим фактором для определения длины СКРС является спад мощности в импульсе накачки. Распространение импульсов в волокне с комбинационным рассеянием (вдоль его оси $z$ ) можно рассмотреть в рамках согласованных уравнений интенсивности накачки $I_{p}$ и стоксовой интенсивности $I_{r}$ следующим образом [12]:

$$
\begin{gathered}
\frac{d I_{r}}{d z}=g_{R} I_{p} I_{r}-\alpha_{r} I_{r}, \\
\frac{d I_{p}}{d z}=-\frac{\omega_{p}}{\omega_{r}} g_{R} I_{p} I_{r}-\alpha_{p} I_{p} .
\end{gathered}
$$

Здесь $g_{R}$ - коэффициент КРС, связанный с сечением спонтанного КРС, а $\alpha_{r}$ и $\alpha_{p}$ - обратные длины затухания интенсивности стоксового излучения и накачки. Система уравнений позволяет оценить критическую интенсивность $I_{\mathrm{pt}}$, при которой мощность затухания в линии сравнивается с усиленной стоксовой компонентой на конце линии, следующим образом [13]:

$$
I_{\mathrm{pt}} \approx \frac{16}{g_{R} L_{\mathrm{eff}}(L)} .
$$

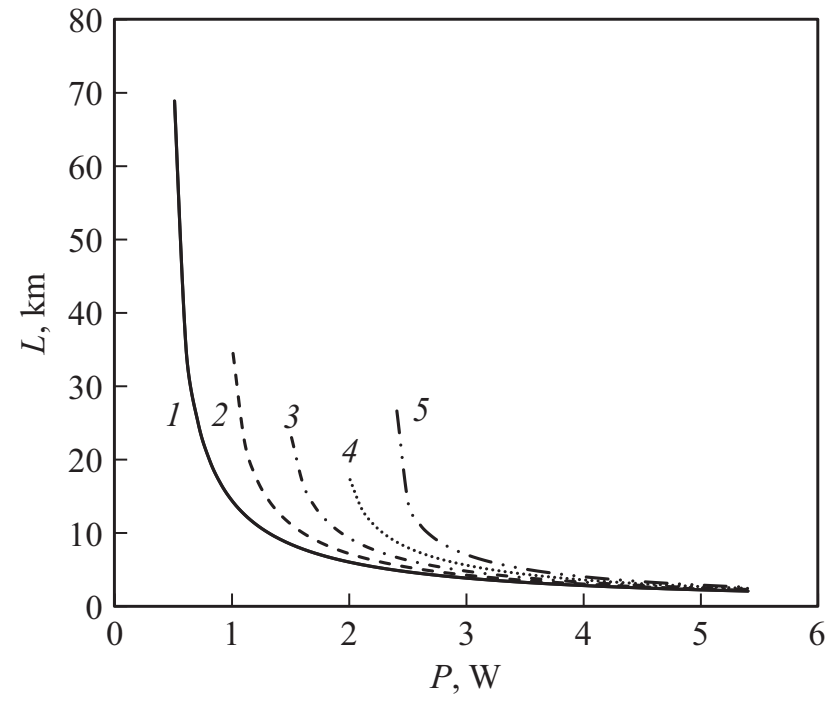

Рис. 1. Зависимость длины линии оптического волокна, в котором комбинационное рассеяние КРС можно рассматривать как усиленное от мощности $P=I_{\mathrm{pt}} S$ импульса накачки. Значения рассчитаны для одномодового оптического волокна площадью поперечного сечения $S=65$ мкм $^{2}$ и разного затухания в волокне 0.2 (1), 0.4 (2), 0.6 (3), 0.8 (4), 1 (5) дБ/км.

Здесь $L_{\text {eff }}$ - эффективная длина оптоволоконной линии, которая отличается от реальной длины $L$ и описывается следующим выражением:

$$
L_{\mathrm{eff}}(z)=\left[1-\exp \left(-\alpha_{p} z\right)\right] / \alpha_{p} .
$$

Выражение (4) было получено в пренебрежении перекачки энергии из импульса накачки в стоксовое излучение, т. е. пренебрегли первым членом в правой части выражения (3). Это приближение оказалось удачным и хорошо описывающим экспериментальную ситуацию [12]. В частности, можно сделать оценку для длины СКРС оптоволоконной линии: комбинируя выражения (5) и (4), можно выразить $L$ через $I_{\mathrm{pt}}$ следующим образом:

$$
L=\frac{1}{\alpha_{p}} \ln \left[\frac{g_{R} I_{\mathrm{pt}}}{g_{R} I_{\mathrm{pt}}-16 \alpha_{p}}\right] .
$$

Значения для длины СКРС оптоволоконной линии, необходимой для эффективного рамановского преобразования, приведены на рис. 1 для разных обратных длин затухания $\alpha_{p}$. Величину стоксовой интенсивности $I_{r}(z)$ в тех же приближениях можно описать следующим выражением:

$$
I_{r}(z)=I_{r 0} \exp \left(g_{R} I_{p 0} L_{\mathrm{eff}}(z)-\alpha_{r} z\right) .
$$

В случае распространения квазиоднофотонных импульсов можно говорить о вероятности (1). При этом можно оценить относительное изменение вероятности КРС $\Delta W_{r} / W_{0}=\left(W_{r}\left(I_{p}\right)-W_{0}\right) / W_{0}$, происходящее из-за добавочного неравновесного числа фононов $n_{r} \sim I_{r}(z)$, 


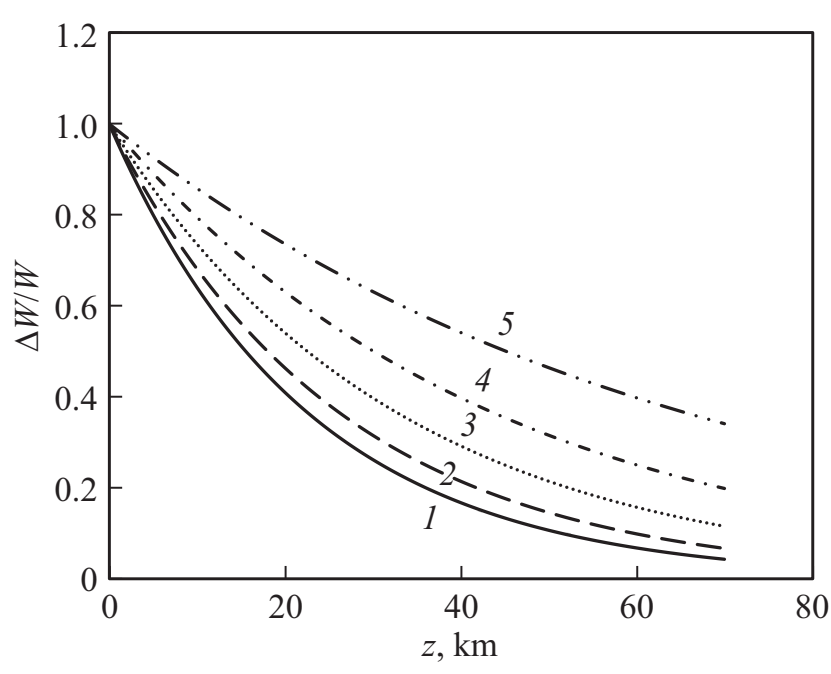

Рис. 2. Относительные изменения вероятности комбинационного рассеяния КРС квазиоднофотонных импульсов в одномодовом оптоволокне после стимулированного комбинационного рассеяния импульса накачки в зависимости от координаты вдоль оптоволокна при разных входных уровнях накачки. Мощность накачки рассматривалиась при поперечном сечении волокна $S=65$ мкм² $^{2}$ и составила 1 (1), 5 (2), 10 (3), 15 (4), 20 (5) Вт.

вызванного КРС импульса накачки. Рассмотрим вероятность рассеяния при учете неравновесных фононов

$$
W_{r} \sim \frac{\omega^{4}(d \varepsilon / d u)^{2}}{4 M N \omega_{0}} \hbar\left(2\left(n_{0}+n_{r}\right)+1\right) .
$$

Тогда с учетом (1) легко получить следующее значение для относительного изменения вероятности рассеяния:

$$
\frac{\Delta W}{W_{0}}=\frac{W_{r}-W_{0}}{W_{0}}=\frac{2 n_{r}}{\left(2 n_{0}+1\right)} \sim I_{r}(z) .
$$

Таким образом, значение относительного изменения вероятности КРС зависит от координаты $(z)$ вдоль оптоволоконной линии и эта зависимость представлена на рис. 2 при разных мощностях накачки.

\section{3. Обсуждение результатов}

Прежде всего стоит обратить внимание, что есть ограничение на мощность излучения, которое легко находится из выражения (6) в виде следующего неравенства:

$$
g_{R} I_{\mathrm{pt}}>16 \alpha_{p}
$$

Это означает существование порога минимальной интенсивности $I_{0}$, с которого процесс стимулированного КРС может стартовать:

$$
I_{0}=16 \alpha_{p} / g_{R}
$$

Исследование порога для стимулированного КРС на сегодняшний день остается недостаточно изученным вопросом, во многом потому, что нет четких критериев для отличия стимулированного рассеяния от спонтанного. Кроме того, пороговый характер стимулированного КРС обычно связывают с пороговым накоплением стоксовых фотонов, что тоже возможно. И в этом смысле исследование КРС ослабленных световых импульсов, сопровождающих импульс накачки с некоторой задержкой, позволит прояснить наличие такого порога. Порог в мощности приведет к пороговой населенности оптических фононов, что будет проявляться в виде порога в вероятности КРС и в пороговой зависимости мощности. Выражение (3) хорошо согласуется с экспериментом, что фактически подтверждает наличие такого порога. Наличие порога так же ожидаемо с точки зрения динамики фононной подсистемы в волокне. Дисперсия оптических фононов довольно слабая, что приводит к увеличению роли взаимодействия между фононами. Взаимодействие фононов, с учетом того что они бозоны, может приводить к таким явлениям, как бозеконденсация, которая, как хорошо известно [14], имеет пороговый характер, как фазовый переход второго рода.

С прикладной точки зрения такая бозе-конденсация оптических фононов будет способствовать резкому увеличению комбинационного рассеяния, что позволит создавать оптические рамановские преобразователи и усилители с уникальными характеристиками.

\section{4. Заключение}

Таким образом, в работе впервые рассмотрено КРС квазиоднофотонных импульсов на неравновесных оптических фононах в одномодовом оптическом волокне с накачкой. Данный процесс можно определить как двухстадийное КРС. Такие двухстадийные методы успешно используются при исследовании полупроводниковых наноструктур. Так как КРС сопровождается понижением частоты (переход в стоксовую моду), данный эффект может быть использован в устройствах понижения частоты фотонов. Такие устройства востребованы в связи с применением однофотонных источников в устройствах квантовой криптографии.

\section{Финансирование работы}

Работа выполнена при финансовой поддержке Министерства образования и науки России в рамках прикладного научного исследования (уникальный идентификатор RFMEFI62419X0052), Соглашение о предоставлении субсидии от 29.11.2020 г. № 05.624.21.0052.

\section{Конфликт интересов}

Авторы заявляют, что у них нет конфликта интересов.

\section{Список литературы}

[1] G. Landsberg, L. Mandelstam. Naturwissenschaften B, 1. 557 (1928).

[2] K.R. Ramanathan. Ind. J. Phys., 2, 387 (1928). 
[3] E. Smith, G. Dent. Modern. Raman spectroscopy - A practical approach (John Wiley \& Sons, LTD, 2005).

[4] E.J. Woodbury, W.K. Ng. Proc. IRE, 50, 2367 (1962).

[5] A.J. Stentz. Proc. SPIE, 91, 3263 (1998).

[6] R.W. Boyd. Nonlinear Optics, 3rd edn (Academic Press, Boston, 2008) Chap. 10.

[7] F.L. Galeener, J.C. Mikkelsen, R.H. Geils, W.J. Mosby. Appl. Phys. Lett., 32, 34 (1978).

[8] К.А. Балыгин, В.И. Зайцев, А.Н. Климов, А.И. Климов, С.П. Кулик, С.Н. Молотков. Письма ЖЭТФ, 105, 570 (2017).

[9] R.R. Alfano. Supercontinum Laser Source: Ultimate White Light (Springer, 2016).

[10] U. Teğin, B. Ortaç. Nature. Sci. Rep., 8, 12470 (2018).

[11] Рассеяние света в твердых телах, под ред. М. Кордона (М., Мир, 1979).

[12] G. Agrawal. Nonlinear fiber optics. 5th edn (Academic Press, Elsiver Oxford, 2013).

[13] R.G. Smith. Appl. Optics, 11, 2489 (1972).

[14] Novel superfluids, ed. by K.H. Bennemann, J.B. Ketterson (University Press, Oxford, 2013).

Редактор А.Н. Смирнов

\section{Raman scattering of quasi-single-photon pulses in pumped fiber}

V.G. Popov 1,2, V.G. Krishtop ${ }^{1,2}$, S.A. Tarelkin ${ }^{3}$, I.I. Korel ${ }^{4}$

${ }^{1}$ Center of scientific research and perspective development, JSC „Infotecs“, 127287 Moscow, Russia

${ }^{2}$ Institute of Microelectronics Technology

Academy of Sciences,

142432 Chernogolovka, Moscow region, Russia

${ }^{3}$ Russian Research Institute

for Optical and Physical Measurements

Federal State Unitary Enterprise,

11936 Moscow, Russia

${ }^{4}$ Novosibirsk State Technical University

(Technical Physics Department),

630073 Novosibirsk, Russia

Abstract Processes of Raman scattering of quasi-single-photon pulses in a single-mode optical fiber with pumping are theoretically considered. The peculiarity of the scattering is that the pumping creates non-equilibrium molecular vibrations, which significantly increases the probability of Raman scattering in the optical fiber. Non-equilibrium vibrations are expected to be when the stimulated Raman scattering takes place for the pump pulse. As a result, the length of the optical fiber has been estimated where the probability of the Raman scattering is increased. 